\title{
Neuropsychological rehabilitation of memory in post-stroke patients with brain nonspecific structure injury
}

\author{
Tatiana Aleksandrovna Mardasova, and Pavel Rafaelevich Iusupov \\ Altai State University, Barnaul, Russia
}

\begin{abstract}
The relevance of the study of the neuropsychological rehabilitation of memory impairments in stroke and the increasing role of the development of the rehabilitation direction to build a competent management system for such patients suggests good conditions for improving the health of the population, increasing the life expectancy and social activity of people.
\end{abstract}

\section{Introduction}

A stroke causes a spectrum of disorders in patients. This leads to disruption of human life, affects the implementation of routine and professional activities, reduces the quality of life of patients. Statistically, memory recovers most effectively in the first three months after a stroke. Therefore, it is important to sufficiently study the problems of post-stroke mnestic disorders $[4,5,8,12,13$, etc. $]$.

In Russia, cerebrovascular diseases rank second among the causes of overall mortality after cardiovascular diseases. About half of all strokes occur under the age of 60 , but every year, according to statistics, it affects younger people. Today, among patients admitted to health care institutions, about $40 \%$ are people of working age $[6,11,14$, etc.]. Memory disorders, as the most common symptoms of strokes, have always been at the center of pathopsychological and neuropsychological research. Russian psychology and neuropsychology have quite well studied the course of mnestic processes, both in normal conditions and in disorders of various origins, and considered the local brain lesions in different types of stroke in detail. For example, A.R. Luria made a great contribution to the study of this problem in his research [10, etc.].

Neuropsychological rehabilitation, corrective measures, and the actual study of changes in the mental activity of patients should be considered as a single sequence of steps starting from the acute post-stroke phase. In this case, one should focus not only on the already formed but also on the expected pathological symptoms. In this regard, correctional work should be preventive. The work of a clinical psychologist in the rehabilitation process begins from the examination and continues until and after the patient. Rehabilitation involves working with the patient, his family, and friends, as well as with a team of specialists who work on patient recovery $[11,16$, etc. $]$. 
Rehabilitation can be defined as a combination of medical, psychological, and social measures aimed at the maximum possible restoration of the physical, psychological, and social status of a person [16].

The objective of the research was to develop and analyze the effectiveness of the program of neuropsychological rehabilitation and recovery of memory in post-stroke patients with brain nonspecific structure injury.

Memory, as the highest mental function, is a set of complex processes that provide a focus on performing the functions of memorizing, preserving, and mentally reproducing previous impressions [10].

Memory impairments are divided into modal-specific and modal-nonspecific disorders $[10,16$, etc.]. Modal-nonspecific memory disorders are manifested as amnestic disorders, amnestic syndrome, or pseudoamnesia (memory disorders as mnestic activity) and characterized by all general types of memory impairment, affecting all modalities.

Modal-specific memory disorders are characterized by violating a specific type of stimuli and one modality - auditory, auditory-speech, or visual. These disorders occur when the analyzer-specific gnostic zones of the cerebral cortex are affected; they are manifested in symptoms of constricted reproduction volume, symptoms of imbalance of traces reflected in paraphases, paragnoses, paragraphs, symptoms of disturbance in maintaining the order of stimuli, contamination, symptoms of retroactive inhibition, reminiscences, etc. $[9$, etc. $]$.

The neuropsychological practical work presented in the article involves the analysis of the effectiveness of diagnostic and rehabilitation measures for stroke in the midline structures of the brain.

Medical diagnosis started with a conversation with the patient, with the help of questions such as "where are you now", "what is your name". Orientation to person, place, and time is determined, as well as spontaneous speech. Also, the study of speech used the repetition of sounds, words, phrases, etc. Vigilance was examined using the methods of Schulte, and the dot cancellation test. In the course of diagnostics, the researchers monitored changes in mental processes during the tests. The study of visual gnosis used tests to recognize various objects, colors, faces, etc. depicted in drawings. For somatosensory gnosis, tests were carried out for the localization of touch, recognition of objects by touch. Auditory gnosis was tested using the assessment and reproduction of rhythms, as well as the localization of sound in space. The study of praxis used constructive, dynamic (fist-rib-palm), motor (drawing a series of symmetrical figures), and other tests. The study of the writing function involved copying a text from the sample, writing letters, various phrases, and sentences. The reading study suggested reading letters, sentences, and texts. The count was checked by solving mathematical problems and producing serial counts (100-7 ...). Memory was assessed using the 10 -word technique, a pictogram, memorization of texts, phrases, and pairs of words read aloud, as well as selfreading. Also, the study of visual memory used various pictures with images of objects and stories. To assess the strength of memory traces under the influence of heterogeneous and homogeneous interference, the subjects were proposed to complete tasks, for example, memorize words, then perform counting tests, and then again recall the words memorized before. Intellectual processes were assessed by understanding stories, plot pictures, as well as solving problems and selecting words by analogy (rose-flower; raspberry-berry) [1, 2, 3, 7,15 , etc.].

The results of the neuropsychological study showed that the patient was reluctant to get in touch during the examination due to constant fatigue, and at some moments fell asleep. Orientation to person, time, and the place was normal. The mood is stable. At the time of the examination, consciousness was clear. 
The subject had difficulties with learning instructions for tasks on the first try; however, with additional explanation and a specific example, he understood instructions but could hardly keep them in work. The general pace of work was reduced, nonuniform, with fluctuations. The patient required frequent breaks for recovery, along with this, low productivity was observed by the end of the examination (which proves that damage to the median structures of the brain reduces performance and causes exhaustion). For the Schulte test, the time the patient spent looking for numbers is above the normal limits; for the rest tables, time is different.

The "Memorizing 10 words" test revealed impairments of direct memorization (memorization curve: $3 ; 5 ; 4 ; 5 ; 6$ ); with delayed reproduction; the patient recalls three words. There is a slow adaptation. The patient was also characterized by an increased reminiscence of traces, that is, with difficulties in recalling words directly, the patient recognizes them in the list with many others after a while. After listening, the patient could reproduce the story only from the second time, and the semantic part - after the leading question.

At the same time, according to the results of the examination, the patient was able to perform tests for visual and auditory gnosis, as well as praxis (which proves that if the median nonspecific structures of the brain are damaged, mental functions of different modalities (vision, hearing) will remain intact). The writing and reading functions were also intact.

The main goal of rehabilitation is the restoration of impaired mental functions due to intact ones, rather than adaptation to the impairment, which is considered in but is not the main activity of the work. Therefore, a rehabilitation program is planned, involving exercises, tasks for each patient depending on the location of the lesion and the personal characteristics of the patient [17].

Neuropsychological rehabilitation and restoration of impaired functions are based on the theory of the structural-functional model of the brain as a substrate of mental activity by A.R. Luria.

The main tasks for memory recovery are to structure information for memorization and reproduction, using the intact functions, include tests aimed at maintaining vigilance, train the patient's mental functions with the involvement of visual and figurative memory, as well as emotionally significant associations and connections when memorizing.

The goals of memory recovery are to reduce the severity of mnestic disorders or prevent their deterioration, reduce the patient's maladjustment by teaching strategies to increase the volume of memorized material, train in compensation strategies (using reminders, notes, short instructions, memory pads), and improve the daily activity and quality of life of the patient.

Based on the results obtained, we draw up a rehabilitation program, which included exercises to restore memory and maintain vigilance.

The developed rehabilitation program consists of 10 sessions, 30-40 minutes each. This program includes tasks and exercises aimed at the rehabilitation and restoration of the cognitive impairment that develops as a result of this syndrome.

We used tasks to restore memory and vigilance. At the same time, to make the rehabilitation program comprehensive, tasks were used to maintain other mental functions. Below is an example of the tasks used in the rehabilitation program:

1. The patient is shown a picture and asked to remember everything depicted on it. After the picture is closed, questions are asked such as "What was shown in the picture?", "What time of year?".

2. The patient is shown a two-column table. The lines contain words (the number of words increases with each session). The right column is closed, and the left one is proposed 
to be remembered. Then the left column is covered, and the patient must circle in the right column the words from the left column.

3. The patient is asked to remember emotionally colored words such as 'house', 'mommy', 'sonny', 'Jack-Frost', 'courtyard' (the number of words increases with each lesson).

4. The patient is shown 10 words on a specific topic to remember with their subsequent reproduction.

5. The patient is shown 10 pictures with objects to remember. Then the patient closes his eyes and one of the pictures changes. The patient must remember which picture is missing.

6. The patient is shown a picture of a tree and pears and apples. Then the patient is asked to paint the apples red and the pears yellow.

During the rehabilitation program, the overall activity increased with each session. The patient solved each new task more willingly. He did his homework after each lesson. Improvements in memory became obvious when the memorized material was organized into semantically meaningful structures. Also, when using a visual analyzer, for example, showing pictures, telling stories, the patient remembered more and later recalled information (which proves that using different modalities (vision, hearing) in a rehabilitation program for restoring memory with damage to the median structures improves its results). Emotionally colored words also helped increase the volume of memorization.

At the final stage of the rehabilitation program, the patient improved his pace significantly. The patient was interested in the program, willingly completed the tasks. Any mistakes made in the tasks were corrected independently, without help.

The repeated neuropsychological study revealed improvements in mental processes. The patient learned the instructions for the tasks mainly at the first try and used them in work. The patient's motivation for recovery increased, so he performed the tasks with due diligence, chirked up when completing them, worked with interest, and tried to find the right solutions and correct mistakes. The pace of work increased but, along with this, signs of moderate fatigue were observed. The time spent on the Schulte test decreased, vigilance became more stable, and there were no significant time differences when performing the test. The volume of direct mechanical memorization has increased and become more productive - the reproduction curve of 10 words was $7,9,10,9,10,10$; delayed - 9 . Thus, the introduction of a mediating link in the memorization process increases its efficiency. At the same time, according to the results of the examination, the patient was able to perform tests for visual and auditory gnosis, as well as praxis; the writing and reading functions were also intact.

\section{Conclusion}

The results of the neuropsychological study show an improvement in memory and voluntary vigilance. They indicate significant improvement in the patient's memory, considering the intact mental functions of perception of different modalities (vision, hearing); adding emotionally colored words also helps increase the memory span.

Based on the results of the study, we can conclude as follows:

1. Damage to the median nonspecific structures of the brain did not affect mental functions of different modalities (vision, hearing).

2. Damage to the median nonspecific structures of the brain reduces working capacity and causes exhaustion. 
3. The use of different modalities (vision, hearing) helped improve the effectiveness of the memory rehabilitation program for patients with damage to the median nonspecific structures of the brain.

4. The use of emotionally colored words, as well as the inclusion of a register of different modalities in neuropsychological rehabilitation due to the presentation of pictures, stories, auditory influences, helped to improve memory in the form of greater memory span and better vigilance.

\section{References}

1. T. V. Akhutina, Clinical and Special Psychology, 2, 1 (2012)

2. E. Iu. Balashova, M. S. Koviazina, Classical stimulus materials (Genesis, 2010)

3. A.P. Biziuk, A compendium of methods of neuropsychological research, 398 (Rech, 2005)

4. E.L. Bragina, Young scientist, 20(100), 115 (2015)

5. V.N. Grigorieva, Cognitive neurorehabilitation of patients with focal brain lesions (MPSI, 2006)

6. V.V. Zakharov, Atmosphere. Neural diseases, 3, 14 (2011)

7. V.V. Zakharov, N.N. Iakhno, Memory impairments, 150 (GeotarMed, 2003)

8. V.V. Zakharov, N.V. Vakhnina, Neurology, neuropsychiatry, and psychosomatics, 2, 8 (2011)

9. N.K. Korsakova, E.Iu. Balashova, Handbook for the diagnosis and treatment of diseases in the elderly, 120 (Novaia Volna, 2000)

10. A.R. Luria, Human higher cortical functions and their disorders in local brain lesions, 378 (2000)

11. V.A. Minenkov, I.R. Shmidt, M.G. Zhestikova, Neuroplasticity, Topical issues of neurology and neurorehabilitation: a collection of scientific papers, 192 (2006)

12. V.A. Parfenov, D.R. Khasanova, Ischemic stroke, 298 (MIA, 2012)

13. I.S. Preobrazhenskaia, N.N. Iakhno, Neurological journal, 45 (2007)

14. S.A. Sidorova, Bulletin of VolSMU, 17, 72 (2006)

15. E.D. Khomskaia, N.Ia. Batova, Neuropsychological study, (MSU Publishing house, 1992)

16. L.S. Tsvetkova, Textbook for university students, 382 (Academic project, 2004)

17. L.S. Tsvetkova, Introduction to neuropsychology and restorative education, 148 (2000) 